\title{
Ten Years Evidence-based High-Tech Acupuncture Part 3: A Short Review of Animal Experiments*
}

\author{
Gerhard Litscher
}

\author{
Research Unit of Biomedical Engineering in Anesthesia and Intensive Care Medicine and TCM Research Center \\ Graz, Medical University of Graz, Auenbruggerplatz 29, A-8036 Graz, Austria
}

\begin{abstract}
High-tech acupuncture research has been performed for 10 years at the Research Unit of Biomedical Engineering in Anesthesia and Intensive Care Medicine at the Medical University of Graz. This article as a part of a series comprises animal experiments in the field of needle and laser acupuncture. The investigations presented in this article were performed in pigs, dogs and sheep. In all studies sedative stimulation effects of the acupoint Yintang are described using different measurement parameters (EEG-bispectral index, EEG spectral edge frequency and metabolic parameters).
\end{abstract}

Keywords: acupuncture - laser acupuncture - traditional Chinese medicine

\section{Introduction}

Methods from complementary medicine are constantly obtaining acceptance in human medicine. Veterinary medicine is also giving more and more attention to these ancient methods (1). After its founding, the exportation of veterinary acupuncture for example from China to Japan, Korea, Europe and North America, led to adaptations unique to each country $(2,3)$.

Besides treatment of animals using acupuncture or acupressure, animal experimental studies have a longstanding tradition in medicine. Along with numerous textbooks and treatment guidelines for veterinary medicine (4-6), there are extensive experimental scientific investigations also in the field of acupuncture. The scientific database 'pubmed' (http://www.pubmed.gov)

For reprints and all correspondence: Prof. Gerhard Litscher, Research Unit of Biomedical Engineering in Anesthesia and Intensive Care Medicine and TCM Research Center Graz, Medical University of Graz, Auenbruggerplatz 29, A-8036 Graz, Austria. Tel: + 43316 385-3907; Fax: + 43316 385-3908; E-mail: gerhard.litscher@meduni-graz.at *This article is part of a presentation which was given by the author as a keynote lecture at the 10th East-West Medical CMIR Conference 'Integrative Medicine-The Future?' in 2007 at the Royal College of Physicians in London $(7,8)$. alone gives 2357 items (January 8, 2008) when searching for 'animal and acupuncture'.

The present third part of a review-series in $e$ CAM $(7,8)$ deals with selected studies describing animal experimental investigations, largely carried out at the Medical University of Graz within the last years. The studies include investigations in pigs, dogs and sheep.

For example, it was investigated within multiparametric biosignal measurements in anesthetized pigs whether effects and possible differences in brain-specific parameters can be found following acupoint stimulation of points Yintang (GV24-2) and Renzhong (GV26), using different stimulation modalities (manual needle acupuncture, laser stimulation) (9).

The aim of another study which was performed in Korea was to evaluate the effect of acupuncture at Yintang or GV20 points on electroencephalographic SEF 95 in dogs (10).

A third study was carried out in sheep, also stimulating the acupoint Yintang (11), and should therefore be mentioned in this article. This study was carried out within a sheep shearing course at the University of Veterinary Medicine in Vienna. The aim of the study was to investigate whether laser acupuncture is able to lessen the increase of the concentration of glucocorticoid metabolites in the feces of the animals after shearing. 


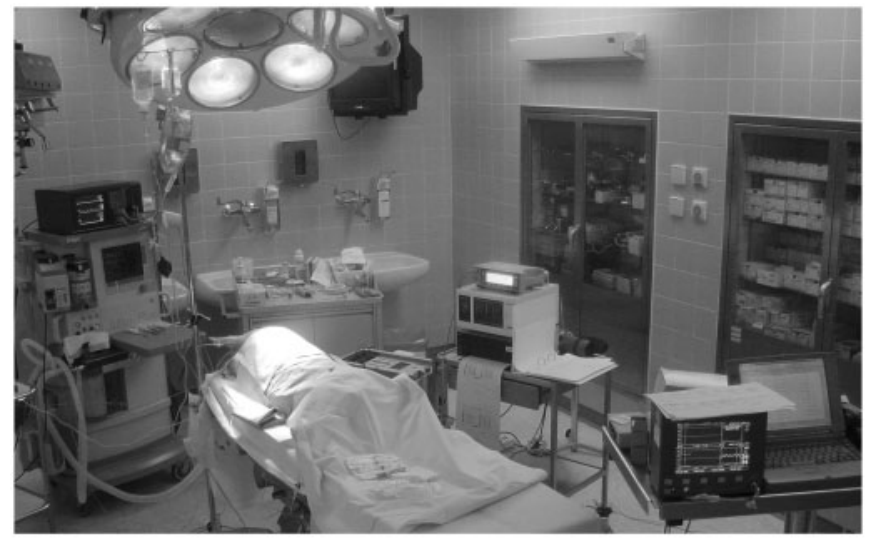

Figure 1. Animal experimental studies at the Medical University of Graz.

\section{Methods}

\section{Yintang-Pigs}

Within this animal experimental study, multiparametric biosignal measurements were carried out before, during and after manual acupuncture needle stimulation and laser stimulation in four pigs (sus scrofa domesticus) (9). The animals were anesthetized in the animal operating room of the Department of Surgical Research at the University Surgical Clinic in Graz (Fig. 1). They had been fasting for the last 24 hours but had access to water. Mean weight $\pm \mathrm{SD}$ was $28.7 \pm 1.2 \mathrm{~kg}$ and the range $28-30 \mathrm{~kg}$. The study was carried out according to the guidelines of the ethical committee.

For premedication the following drugs were used: diacepam (Gewacalm ${ }^{\circledR}$, Nycomed, Linz, Austria), azaperon (Stresnil ${ }^{\circledR}$, Janssen-Cilag Pharma, Vienna, Austria) and ketamin $\left(\mathrm{Ketasol}^{\circledR}\right.$, aniMedica, Senden-Bösensell, Germany). Induction of anesthesia in the test animals was performed intravenously with fentanyl, propofol (Diprivan ${ }^{\circledR}$, Astra Zeneca, Vienna, Austria) and rocuronium (Esmeron ${ }^{\circledR}$, Organon, Oss, Netherlands). Maintenance of anesthesia was done with propofol (Diprivan ${ }^{\circledR}$, Astra Zeneca, Vienna, Austria), remifentanyl (Ultiva ${ }^{\circledR}$, Glaxo Smith Kline Pharma, Vienna, Austria) and rocuronium (Esmeron ${ }^{\circledR}$, Organon, Oss, Netherlands). The exact doses are described in the previous original publication (9).

The study was carried out as a randomized, controlled cross-over trial. The order of stimulation conditions (needle acupuncture at Yintang, laser stimulation at Yintang, needle acupuncture at Renzhong and needle acupuncture at a control point) was randomized, and the interval between the test trials was at least $5 \mathrm{~min}$.

The measurement data were registered while the animals were anesthetized. The following measurement readings were analyzed ( $a=2 \mathrm{~min}$ before stimulation; $b=$ during stimulation; $c=2 \mathrm{~min}$ after end of stimulation).

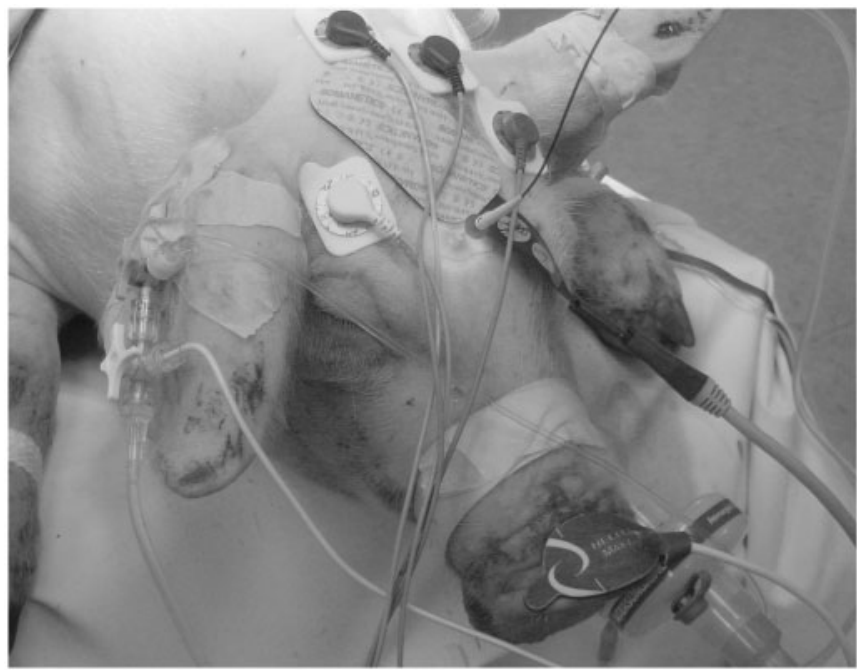

Figure 2. EEG and oxygen saturation recording in a pig. In this case the acupoint Yintang is stimulated with an optical needle.

According to Traditional Chinese Medicine, the acupoint Yintang is said to have sedating effects in human medicine, whereas Renzhong is said to have stimulating effects (12).

Manual needle acupuncture was carried out using singleuse sterile needles of $0.30 \times 30 \mathrm{~mm}$ (Huan Qiu, Suzhou, China). Needling was vertical $(0.5 \mathrm{~cm})$. Stimulation (for $20 \mathrm{sec}$ ) was performed in $1.5 \mathrm{~min}$ intervals, consisting of a combination of rotating and thrusting movements. After a total of $5 \mathrm{~min}$ the needle was removed.

Laser stimulation is an optical, pain-free stimulation method (12-14). The optical needle used (wavelength: $685 \mathrm{~nm}$; diameter: $500 \mu \mathrm{m}$; time of irradiation: $5 \mathrm{~min}$; energy density: $\sim 1.2 \mathrm{~kJ} \mathrm{~cm}^{-2}$ ) emits red light in a continuous wave mode with an output power of $30-40 \mathrm{~mW}$.

Bioelectrical brain activity (EEG) was recorded continuously using an Aspect A-1000 System (Aspect Medical Systems Inc., Natick, MA, USA). Four EEGelectrodes were applied to the skull (comp. Fig. 2). The two channels of spontaneous bioelectrical brain activity were recorded using Zipprep-electrodes (Aspect Medical Systems Inc., Natick, MA, USA). The electrode impedances were $<3 \mathrm{kOhm}$. The low cutoff frequency was $2 \mathrm{~Hz}$ and the high cutoff frequency $30 \mathrm{~Hz}$. Bispectral index (BIS) was analyzed (12).

Together with the multiparametric brain function measurement signals, additional parameters were recorded: heart rate and peripheral oxygen saturation on the upper side of the snout (Oximax, Nellcor N-595, Tyco Healthcare Group, Pleasanton, USA; comp. Fig. 2); peripheral oxygen saturation at the tail stump; blood pressure in the left femoral artery (Cardiocap, Datex, Hoevelaken, Netherlands) and regional cerebral oxygen saturation (INVOS 5100, Somanetics, Troy, MI, USA). 


\section{Yintang-Dogs}

Twelve healthy intact male miniature schnauzer dogs $(4.2 \pm 6.1 \mathrm{~kg}, \quad 1-2$ years old) without neurological abnormalities and disease were investigated in this study (10). The study adhered to the strict guidelines of the 'Guide for the Care and Use of Laboratory Animals' of Seoul National University.

The Yintang and GV20 acupoint were used. Dogs were assigned randomly to 10 experimental groups $(n=5$ per group) and 5 control groups ( $n=5$ per group) (10).

Different experimental drugs were used: acepromazine (Sedaject $^{\circledR}$, Samu median Co., LTD, Seoul, Korea), butorphanol (Butophan ${ }^{\circledR}$, Myung moon pharm Co., LTD., Seoul, Korea), diazepam (Melode ${ }^{\circledR}$, Dong whap arm. IND. Co., LTD, Seoul, Korea), medetomidine (Domitor ${ }^{\circledR}$, Orion pharma, Finland) and Phenobarbital (Luminal $^{\circledR}$, Dai han pharm. Co. LTD., Seoul, Korea) (10). For the exact doses see again the original publication (10). The EEG electrode arrangement consisted of placing eight electrodes on the head, a reference electrode at the right ear and a ground electrode at the left ear (for the exact positioning please refer to the original publication).

The SEF 95 is defined as the 95th percentile of the power distribution. This usually is the frequency which represents $95 \%$ of the total power. It is calculated by using the area under the curve of the power spectrum. High SEF 95 values occur in the awake state, while low SEF 95 depicts a depressed cerebral activity which corresponds with deep sleep or deep anesthesia. Further methodological aspects of the study can be found in the original paper (10).

\section{Yintang-Sheep}

The test animals were 77 adult sheep (age: 1-7 years).

Laser acupuncture at acupoints Yintang, GV20, bilateral: St.36, Sp.6 and UB.23 was performed using a $50 \mathrm{~mW}$ diode-laser (diameter: $6.2 \mathrm{~mm}$ ) with a 'hidden monitor system'. The selected points were illuminated with an energy of $1 \mathrm{~J} \mathrm{~cm}^{-2}$ (11).

In the control group, the laser was also activated and held to the skin for exactly $20 \mathrm{sec}$, but the laser beam was completely shielded by applying an insulation cap on the tip. Fecal samples were taken directly from the rectum. The first sample was taken immediately after shearing and served as basal value. Further samples were taken 6, 8, 10, 12 and 14 hours later in order to detect the maximum increase in glucocorticoid metabolite concentration (11).

\section{Results}

\section{Yintang-Pigs}

When stimulating the acupoint Yintang, insignificant decreases of the BIS values can be observed; when
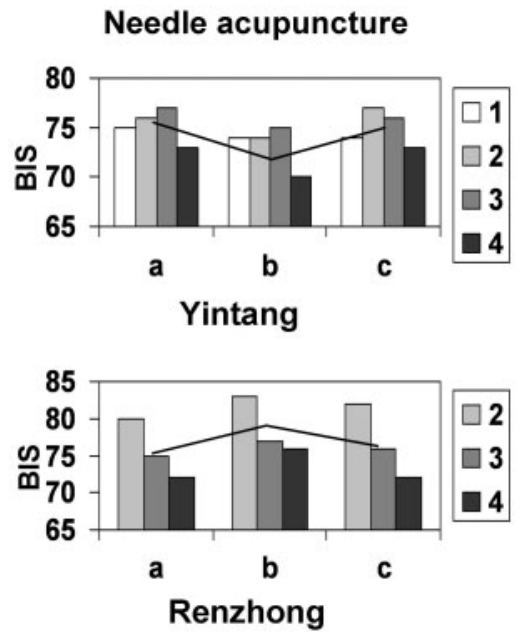

Figure 3. Comparison of effects of manual needle stimulation (a: before; b: during; c: after stimulation) at the acupoints Yintang (top) and Renzhong (bottom) in anesthetized pigs (white column: pig \#1, light gray: pig \#2, medium-dark gray: pig \#3, dark gray: pig \#4). Note the different reactions (decreases and increases of bispectral index (BIS)). Modified from (9).

needling the acupoint Renzhong, the index increases markedly. This trend is illustrated by Fig. 3 (9).

\section{Yintang-Dogs}

When the subjects were conscious, the baseline SEF 95 values recorded in all groups were shown to be similar (10). After the administration of sedatives, the mean SEF 95 values $( \pm$ SD) significantly decreased when compared with the SEF 95 values for a conscious state.

After acupuncture at the Yintang or GV20 point, the mean SEF 95 values significantly decreased in the butorphanol group when compared with those taken following administration of sedatives $(\mathrm{P}<0.05)$. These values also showed a significant difference in contrast to the SEF 95 values of the control group. In contrast, the mean SEF 95 values in other groups did not show any difference after the administration of acupuncture at the GV20 and Yintang points.

\section{Yintang-Sheep}

Six hours after the stress of shearing, the control group which had not been treated with laser stimulation showed a significant increase of the concentration of glucocorticoid metabolites (median: $64.9 \mathrm{ng} \mathrm{g}^{-1}$ feces). The verum group on the other hand showed no significant increase of the concentration of glucocorticoid metabolites (median: $6.6 \mathrm{ng} \mathrm{g}^{-1}$ feces) (11). When comparing the concentration of glucocorticoid metabolites after 8, 10, 12 and $14 \mathrm{~h}$, both groups showed a significant increase and therefore no difference between animals which had been treated and those which had not (11). 


\section{Discussion}

The acupoint Renzhong (GV26) is one of the most often used points of Traditional Chinese Medicine in emergency medical aid and resuscitation $(9,12,15-17)$. In animals, this point is found in the centre of the horizontal line connecting the lower rim of the nostrils $(15,18)$. According to literature, intensive needling of this point can cause restoration of respiration in $90-100 \%$ after phases of apnea within 10-30s (19). According to the same authors, successful resuscitation following cardiac arrest can be achieved in $40-50 \%$ if stimulation is carried out for 5-10 min (19). Amongst other researchers, Chan et al. (15) also conclude that animals can be resuscitated effectively by acupuncture at acupoint GV26 in cases of an overdose of anesthetics, apnea and also cardiac arrests.

In our investigations in pigs needle stimulation of GV26 was able to increase the BIS value from averaged 75.7 to $78.3(+2.6)$ under constant conditions of anesthesia (9). Under almost identical conditions, stimulation of Yintang reduced the mean BIS values from 75.3 to $73.3(-2.0)$. It was therefore shown that the two acupuncture points induce different effects in the bioelectrical activity of the brain (bispectral index). While acupoint GV26 has effects similar to an arousal reaction, needling and stimulation of acupoint Yintang rather causes a deactivation and moderation of bioelectrical brain activities (20). This could be shown in several studies in humans, by our research group (21) as well as by other authors (22).

Recent results from Korea, partly published together with our research group, show that similar effects can also be scientifically traced in dogs $(10,23)$. In one study (10), we examined the sedative effect induced by acupuncture, at Yintang and GV20 points, when combined with sedatives using different mechanisms. It was found that SEF 95 values decreased the most when combined with the butorphanol. From this result, it is suggested that a combination of acupuncture and opioids might produce the best sedative effect (10).

Concerning the measurements in sheep the authors (11) stated that despite the significant difference between the verum group and the control group which showed after $6 \mathrm{~h}$, no definite conclusion can be drawn concerning the effect of laser acupuncture on an increase of concentration of glucocorticoid metabolites in the feces of sheep (11). Possible reasons for this result may include the low dose and short time of laser stimulation. Repeated laser acupuncture (before and after shearing) might shed light on the question if the effects of laser are only of short duration. Another important factor for further investigations could be the habituation of the sheep to the necessary manipulations in order to minimize stress before acupuncture (11).
In conclusion, the partly placebo-controlled, randomized cross-over animal experimental pilot studies showed that needling and laser stimulation of the acupoint Yintang and other points can induce effects on parameters of bioelectrical brain activity (bispectral index, SEF 95), whereas the stimulation of control points did not yield significant changes. This is in accordance with studies carried out in humans $(21,22)$.

\section{Acknowledgements}

The author thanks Lu Wang for performing acupuncture and Ingrid Gaischek for her valuable support in data registration and analysis (both Research Unit of Biomedical Engineering in Anesthesia and Intensive Care Medicine and TCM Research Center Graz, Medical University of Graz). The author also thanks Prof G. Schwarz, W. Nemetz, F. Kaltenböck and D. Nagele-Moser, from the Medical University of Graz (9). The research was supported partly by the Zukunftsfonds of the Styrian Government (project 4071) and 3B Scientific GmbH, Hamburg.

\section{References}

1. Schoen AM, Wynn SG (Hrsg.). Naturheilverfahren in der Tiermedizin. Grundlagen und Praxis der Klein- und Großtierbehandlung. München: Elsevier Urban \& Fischer, 2005.

2. Jaggar DH, Robinson NG. History of veterinary acupuncture. In: Schoen AM (ed). Veterinary Acupuncture, Ancient Art to Modern Medicine, 2nd edn. St. Louis: Mosby, 2001, 13.

3. Robinson NG. Veterinary acupuncture. An ancient tradition for modern times. Altern \& Complement Ther 2007;13:259-65.

4. Schoen AM (Hrsg.). Akupunktur in der Tiermedizin. München, German: Elsevier Urban, \& Fischer, 2003.

5. Schoen AM, Wynn SG. Naturheilverfahren in der Tiermedizin. München: Elsevier Urban and Fischer, 2004.

6. Wynn SG, Marsden S. Leitfaden Naturheilverfahren in der Kleintierpraxis. München: Elsevier Urban, \& Fischer, 2005.

7. Litscher G. Ten years evidence based high-tech acupuncture - a short review of centrally measured effects. Evid Based Complement Alternat Med 2007, published online Nov 12; doi:10.1093/ecam/ nem 169.

8. Litscher G. Ten years evidence based high-tech acupuncture - a short review of peripherally measured effects. Evid Based Complement Alternat Med 2007, published online Nov 12; doi:10.1093/ecam/nem 145 .

9. Litscher G. Animal experiment investigations and acupunctureYintang and Renzhong. Schmerz \& Akupunktur 2007;1:22-7.

10. Kim MS, Soh KS, Nam TC, Seo KM, Litscher G. Evaluation of sedation on electroencephalographic spectral edge frequency 95 in dogs sedated by acupuncture at GV20 or Yintang and sedative combination. Acup Electrother Res 2006;31:201-12.

11. Lexen E, Nissel H, Gaggl G, Grohmann KH. Can laser acupuncture reduce the increase of glucocorticoid metabolities in the faeces of sheep after mental stress? Dt Ztschr $f$ Akup 2007;50:6-12.

12. Bahr F, Bushe-Centmayer K, Dorfer L, Jost F, Litscher G, Suwanda S, et al. (Hrsg.). Das große Buch der klassischen Akupunktur. München Jena: Elsevier Urban \& Fischer, 2006.

13. Litscher G, Schikora D (eds). Laserneedle-Acupuncture. Science and Practice. Lengerich Berlin Bremen Miami: Pabst Science Publishers, 2005.

14. Litscher G, Schikora D (Hrsg.). Lasernadel-Akupunktur. Wissenschaft und Praxis. Lengerich Berlin Bremen: Pabst Science Publishers, 2004. 
15. Chan WW, Chen KY, Liu H, Wu LS, Lin JH. Acupuncture for general veterinary practice. J Vet Med Sci 2001;63:1057-62.

16. Zhang C. The brain-resuscitation acupuncture method for treatment of post wind-stroke mental depression - a report of 45 cases. $J$ Tradit Chin Med 2005;25:243-6.

17. Zhang M, Zhang H, Liu L. The study of effect of pain in the change of respiration by stimulating "renzhong" acupoints. Zhen $\mathrm{Ci}$ Yan Jiu 1990;15:147-49.

18. Rogers PAM, Skarda R. Emergency acupoint Renzhong (GV26): a bibliography and review from textbook sources. In: Proceedings of the 25th Annual International Congress on Veterinary Acupuncture. International Veterinary Acupuncture Society: Lexington, Kentucky, USA, 1999, 83-9.

19. Janssens LAA, Altman S, Rogers PAM. Respiratory and cardiac arrest under general anaesthesia: treatment by acupuncture of the nasal philtrum. Vet Rec 1979;105:273-6.
20. Weixler D. Sedierungszustände - Sedierungseffekte. In: Weixler D, Paulitsch K (eds). Praxis der Sedierung. Wien: Facultas Universitätsverlag, 2002, 21-38.

21. Litscher G. Effects of acupressure, manual acupuncture and Laserneedle ${ }^{\circledR}$ acupuncture on EEG bispectral index (BIS) and spectral edge frequency (SEF) in healthy volunteers. Europ $J$ Anaesthesiol 2004;21:13-9.

22. Paraskeva A, Melemeni A, Petropoulos G, Siafaka I, Fassoulaki A Needling of the extra 1 point decreases BIS values and preoperative anxiety. Am J Chin Med 2004:32:789-94.

23. Kim MS, Nam TC. Electroencephalography (EEG) spectral edge frequency for assessing the sedative effect of acupuncture in dogs. J Vet Med Sci 2006;68:409-11.

Received January 10, 2008; accepted April 8, 2008 


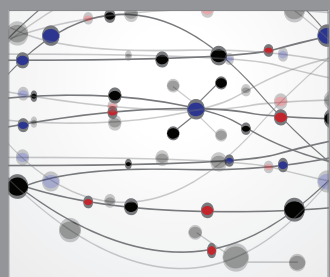

The Scientific World Journal
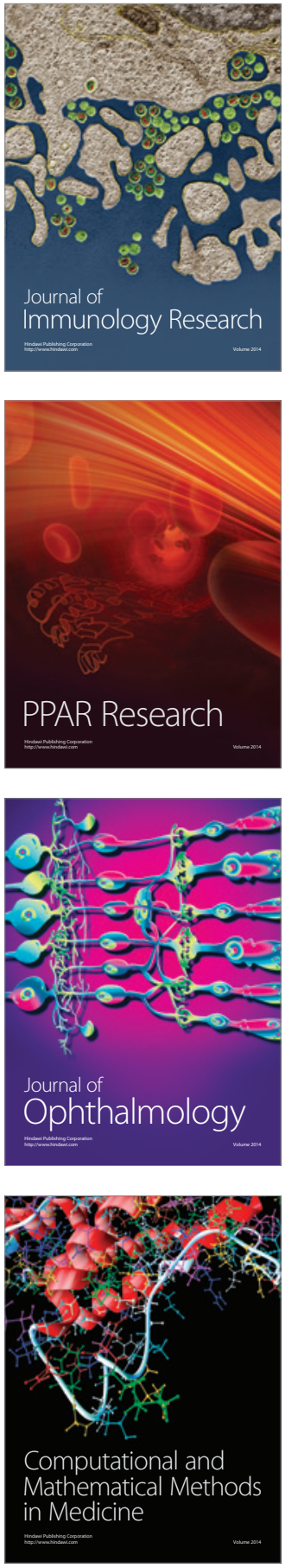

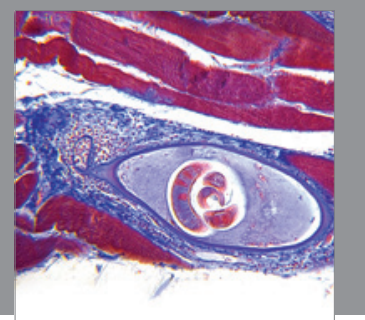

Gastroenterology

Research and Practice
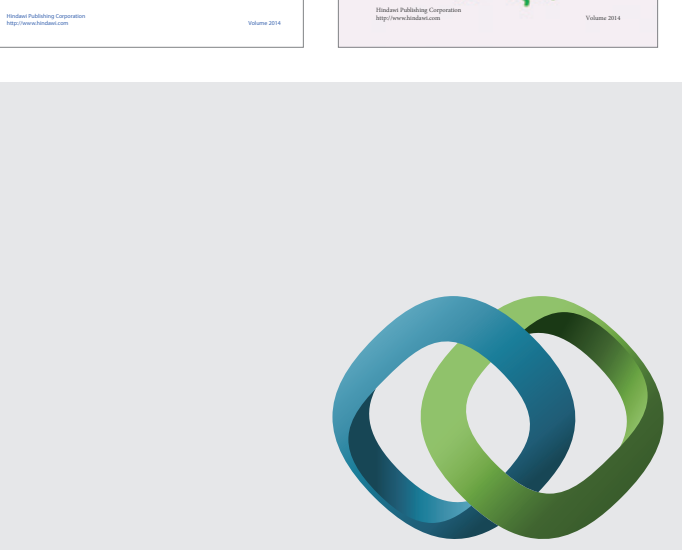

\section{Hindawi}

Submit your manuscripts at

http://www.hindawi.com
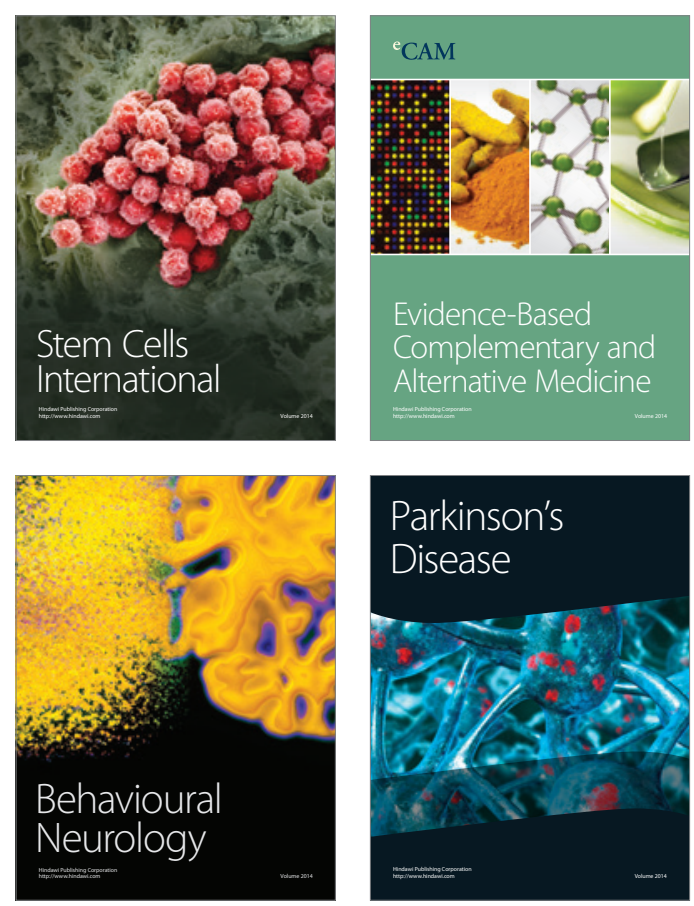

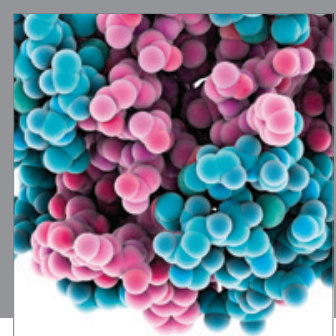

Journal of
Diabetes Research

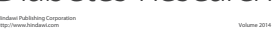

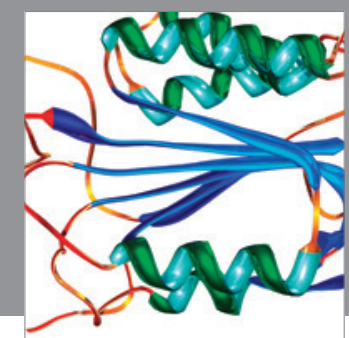

Disease Markers
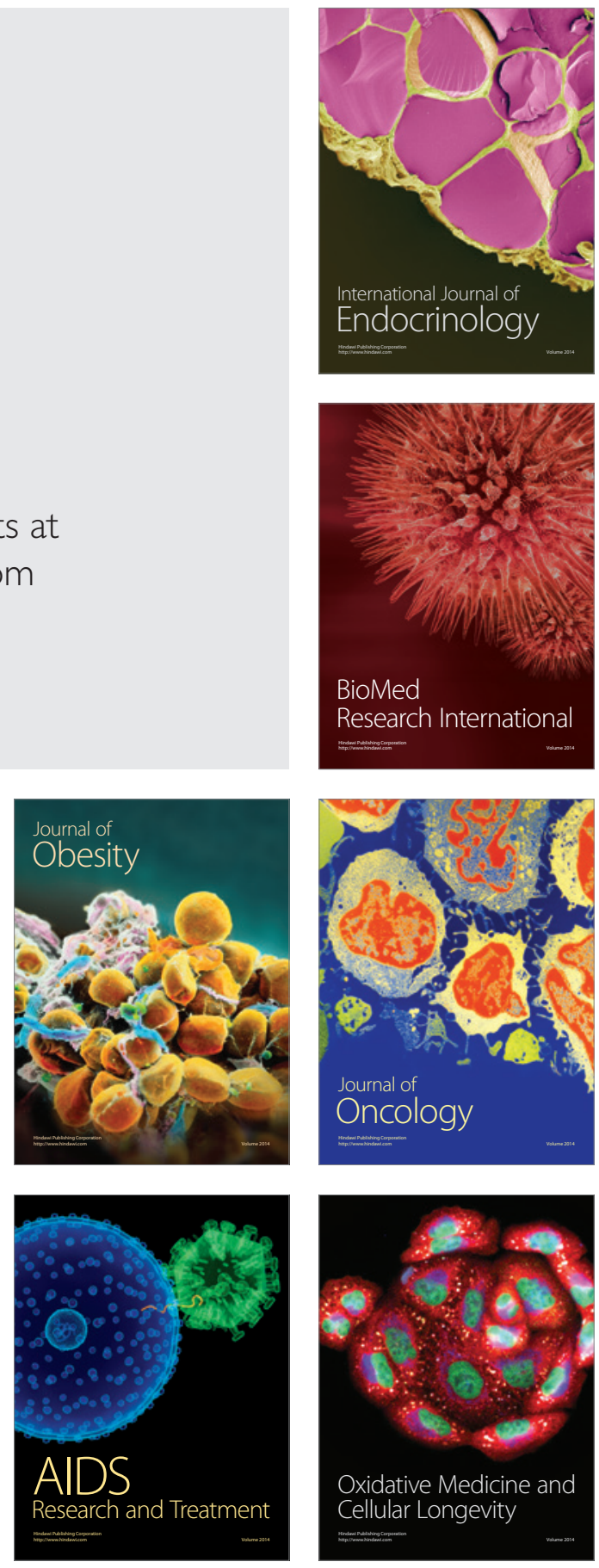